\title{
Comparative evaluation of the efficacy of glycine powder air polishing with scaling and root planing and with fluoride prophypaste as an adjunctive to surgical periodontal therapy- A clinical study
}

\author{
Ayushi Sharma, ${ }^{1, *}$, Meera M Nair ${ }^{2}$, Maydina Achom ${ }^{3}$, Chandan Pal $^{4}$, Mallika Sethi ${ }^{5}$, Nikhil Sharma ${ }^{6}$ \\ ${ }^{1-4}$ Post Graduate Student, ${ }^{5}$ Associate Professor, ${ }^{6} \mathrm{HOD}$, Dept. of Periodontology and Oral Implantology, I.T.S Centre for Dental \\ Studies \& Research, Muradnagar, Ghaziabad, Uttar Pradesh, India
}

*Corresponding Author:

Email: drayushisharma26@gmail.com

\begin{abstract}
Introduction: The use of the glycine powder air-polishing (GPAP) technique may simplify periodic subgingival instrumentation and may be an alternative to the conventional techniques of subgingival biofilm removal. This study aimed to investigate the efficacy of glycine powder air polishing as an additional approach to non surgical periodontal therapy in moderate to deep periodontal pockets, with regard to its clinical effectiveness and bactericidal abilities.

Materials and Methods: A total of 20 sites with moderate to deep periodontal pockets were included in the study. The subjects were allocated into two groups viz. the test group and the control group randomly using toss of a coin. 10 sites each with moderate to deep periodontal pockets were treated with scaling and root planing + glycine powder air polishing (Test Group) and scaling and root planing + prophyhpaste (Control Group). Clinical parameters such as gingival index (GI), plaque index (PI), pocket probing depth were recorded (PPD).

Results: In test group PI, GI, PPD significantly reduced after 21 days follow-up compared to baseline. Pocket probing depth reduced from 5-7 mm at baseline to approx. 1-2 mm at 21 days. Similarly, PI, GI, PPD \& CAL significantly reduced in control group also after 21 days follow-up compared to baseline. Pocket probing depth reduced from 5-7 mm at baseline to approx. 3-4 $\mathrm{mm}$ at 21 days.

Conclusion: It is concluded that GPAP, as an additional approach to nonsurgical periodontal treatment, may be beneficial in the short-term improvement of subclinical periodontal inflammation and it was effective in decreasing inflammation, and pocket probig depth in chronic periodontitis patients and can be successfully used as an adjunct to SRP in routine to non-surgical periodontal therapy.
\end{abstract}

Keywords: Glycine powder air polishing, Prophypaste, Non-surgical periodontal therapy, Biofilm, Bactericidal.

\section{Introduction}

The primary goal for periodontal therapy and periodontal maintenance therapy (PMT) is Supra and Subgingival biofilm. For debridement purposes, hand instruments, sonic or ultra-sonic scalers may be used. The use of these instruments is technically demanding, and if debridement is performed periodically in PMT clinically relevant tooth substance loss may occur in the course of time. ${ }^{1}$ Therefore, the use of the glycine powder air-polishing (GPAP) technique may simplify periodic subgingival instrumentation and may be an alternative to the conventional techniques of subgingival biofilm removal.

Main cause of gingivitis and periodontitis are associated with the accumulation of bacteria on tooth surfaces. Thus, regular mechanical removal of bacterial plaque from all non-shedding oral surfaces is considered the primary means to prevent and stop the progression of periodontal disease. Combination of scaling and root planing is the standard treatment approach.

Subgingival debridement, performed along with supragingival debridement, is considered essential in treating periodontitis. Repeated subgingival debridement as part of supportive periodontal therapy is necessary for removal of biofilm formation to maintain long term periodontal health and to prevent the further loss of periodontal attachment in patients with periodontitis. During supra and subgingival debridement for thorough removal of the biofilm the GPAP is directed approximately at an angulation of 60 90 degree to the long axis of the root. Allowing parts of the jet stream of the injection abrasive water jet device show inevitably affect on the gingival epithelium surrounding the tooth.

With the goal of establishing an efficient and safe technique for subgingival biofilm removal in SPT, a low-abrasive glycine powder was developed for use in commercially available injection abrasive water jets, also known as air polishing devices. ${ }^{2}$ The abrasiveness of glycine powder is one fifth less as compared to bicarbonates. However, it does not seem to have any discernible effect on calculus. The root surface damage is considerably lower after glycine powder air polishing (GPAP) as compared to sonic, piezoelectric and magnetostrictive ultrasonic scalers. In addition, sodium bicarbonate air polishing may cause erosive change to gingival epithelia. ${ }^{3}$ Two clinical studies in patients undergoing supportive periodontal therapy have shown that GPAP is effective (and even superior to curettes) for removal of subgingival plaque in periodontal pockets and it is indeed more comfortable than hand 
instrumentation, as reported by patients. ${ }^{4}$ With increasing pocket probing depth (PPD), however, a greater proportion of subgingival root surfaces remained covered with biofilm. ${ }^{5}$

Furthermore, the risk of excessive root substance removal by GPAP is minimal when compared to root instrumentation using hand instruments or sonic or ultrasonic scalers. ${ }^{6-10}$

The primary objective of this clinical trial is to compare and evaluate the efficacy of GPAP with SRP and with fluoride prophypaste as adjunctive to surgical periodontal therapy in periodontal pockets with PDs of 4 to $9 \mathrm{~mm}$.

\section{Materials and Methods}

A randomized controlled clinical trial study was carried out on the subjects attending the outpatient clinic of the dept. of periodontology and oral implantology, ITS centre for dental studies and research, Muradnagar-Ghaziabad, UP having moderate to deep periodontal pockets. The subjects were selected from the individuals scheduled for routine oral examination. A total of 20 sites with moderate to deep periodontal pockets were included in the study. The subjects were allocated into two groups viz. the test group and the control group randomly using toss of a coin. 10 sites each with moderate to deep periodontal pockets were treated with scaling and root planing + glycine powder air polishing (Test Group) and scaling and root planing + prophyhpaste (Control Group). (Fig. 2)

Inclusion criteria for the study are as follow: 1) 1855 years of age, systemically healthy and without any known conditions or disorders. 2) A diagnosis of chronic periodontitis, having atleast 20 teeth present excluding third molars. 3) The presence of atleast 2 teeth with probing depth $>5 \mathrm{~mm} \mathrm{4}$ ) $\mathrm{mm}$ in 2 quadrants. Exclusion criteria includes 1) pregnancy or lactating women. 2) Allergy to glycine powder 3) systemic medical conditions requiring antibiotic prophylaxis before dental procedures 4) systemic infectious disease 5) smoking more than five cigarettes per day 6) signs of generalized severe periodontitis 7) having received periodontal maintenance within 3 months before baseline 8) teeth with $\mathrm{PD}$ of $\geq 10 \mathrm{~mm}$; 9) teeth having $<3 \mathrm{~mm}$ residual alveolar bone level as assessed on radiographs; or 10) molars showing Class III furcation invasion.

Test group: Periodontal maintenance therapy was performed by air polishing using a fine-grain glycine powder. Periodontal pockets with PD of 4 to $9 \mathrm{~mm}$ at screening were used as investigational sites. Sub gingival biofilm at investigational sites was removed by subgingival GPAP using an air-polishing device with a hand piece equipped with a thin, wedge-shaped nozzle (Fig. 1). The nozzle was inserted gently at an angulation of 60-90 degree into the periodontal pocket until resistance was felt. After activation of the air- polishing device, the nozzle was moved over the entire subgingival root surface using a circular motion. Each tooth surface (mesial, buccal, distal, and lingual) was treated for 5 seconds.

Control group: Periodontal maintenance therapy procedure was performed same as the test group but using polishing paste (prophypaste) instead of GPAP. Two different examiners conducted the study. The first examiner was the clinical operator who conducted each type of treatment. The second examiner recorded the data who was blinded to the treatment received by patients. The clinical parameters like Gingival index (Loe and Silness), ${ }^{11}$ Turesky-Gilmore-Glickman Modification of Quigley Hein plaque index, ${ }^{12}$ PPD (measured using a UNC 15 probe) were recorded at baseline, 7 days, 14 days and 21 days.

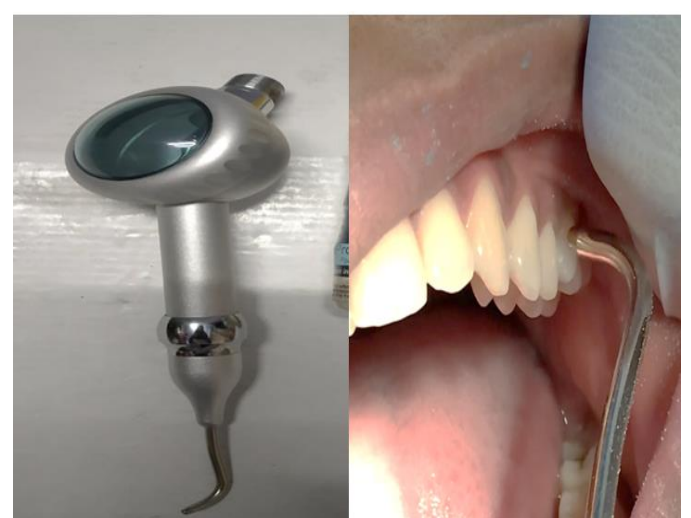

Fig. 1: Air Polisher with nozzle

\section{Statistical Analysis}

The Statistical Analysis Software (SPSS version 16) was used for data processing and analysis. The differences in means of the parameters at the baseline between test and control groups were evaluated using an independent t-test. The changes in parameters over time were evaluated using a paired t-test for each group separately, $\mathrm{p}<0.05$ was considered statistically significant.

\section{Results}

In this randomized controlled clinical trial, there was improvement of the recorded parameters (PI, GI, CAL, PPD) in both the groups (test \& control), with no adverse effects.

In test group PI, GI, PPD significantly reduced after 21 days follow-up compared to baseline. Pocket probing depth reduced from $5-7 \mathrm{~mm}$ at baseline to approx. 1-2 mm at 21 days. Similarly, PI, GI, PPD \& CAL significantly reduced in control group also after 21 days follow-up compared to baseline. Pocket probing depth reduced from $5-7 \mathrm{~mm}$ at baseline to approx. 3-4 mm at 21 days. (Fig. 3, 4, 5)

On inter group comparison between test \& control group, there was no statistically significant difference amongst the clinical parameters (PI, GI, PPD, \& CAL) 
at baseline. However, after 4 weeks follow-up the test recorded parameters compared to control group. group showed statistically significant improvement in

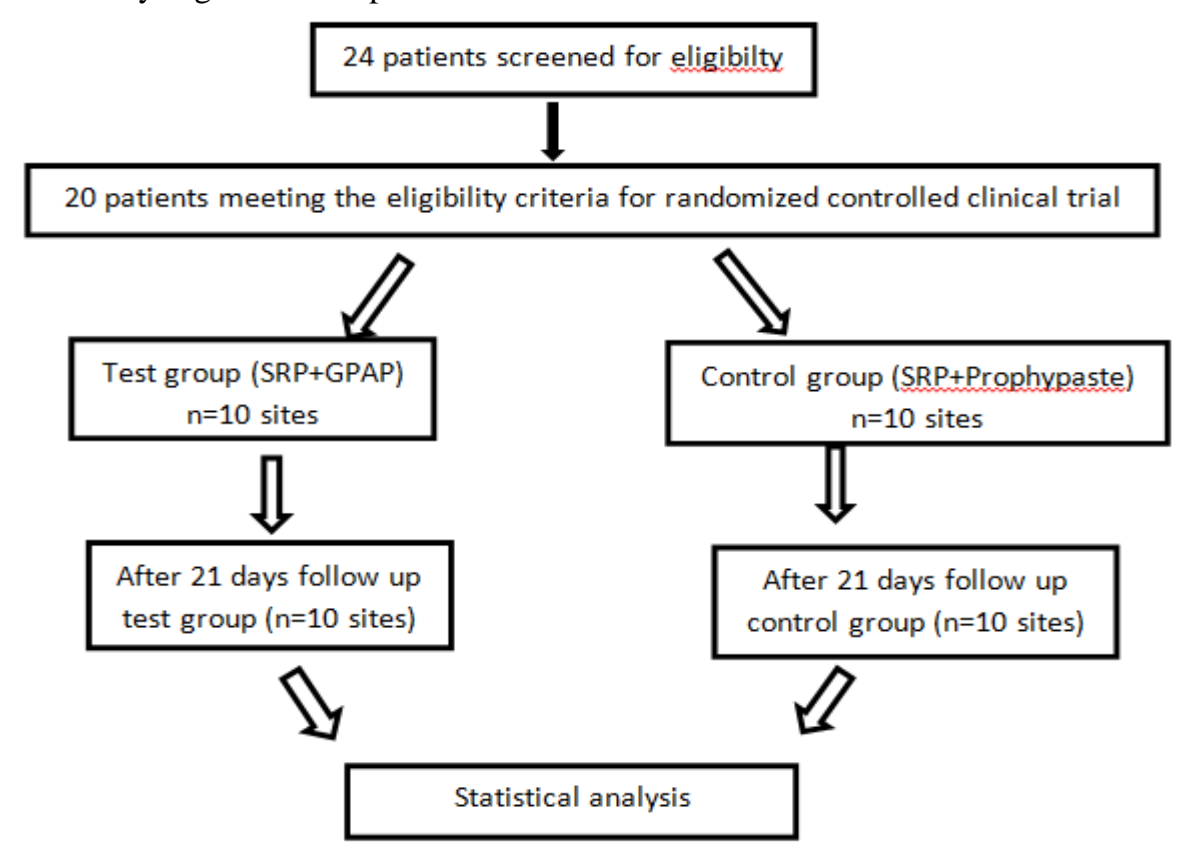

Fig. 2: Study design

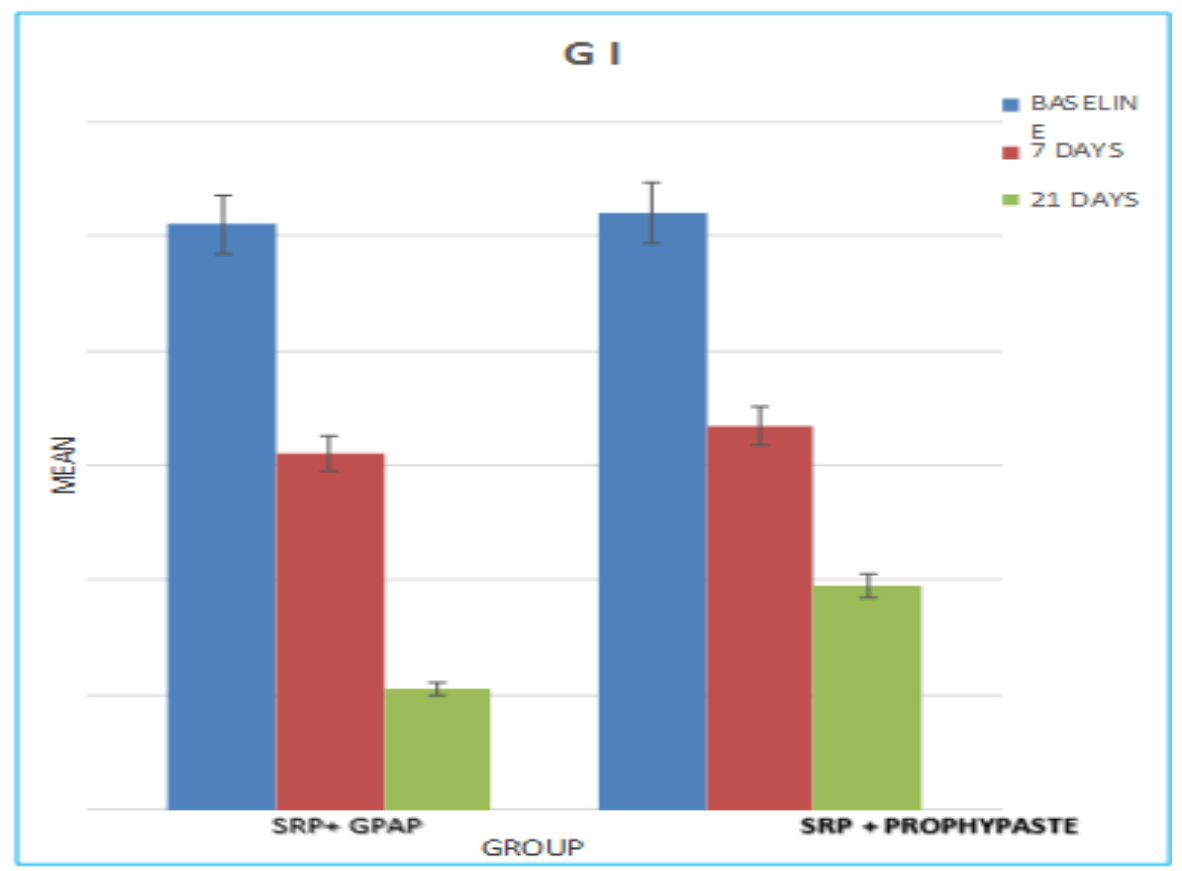

Fig. 3: Graph showing comparison of gingival index in test and control group 


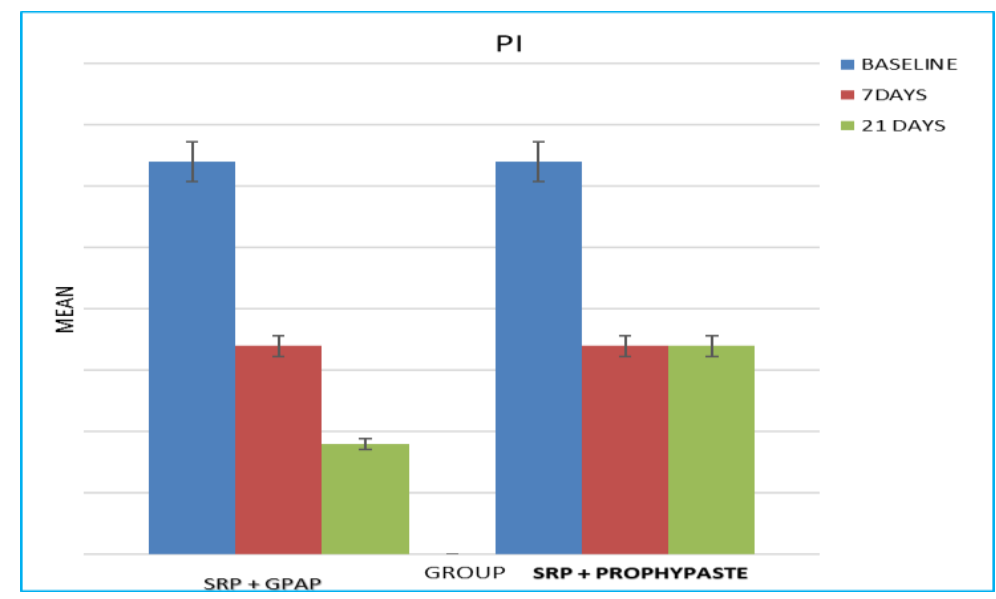

Fig. 4: Graph showing comparison of plaque index in test and control group

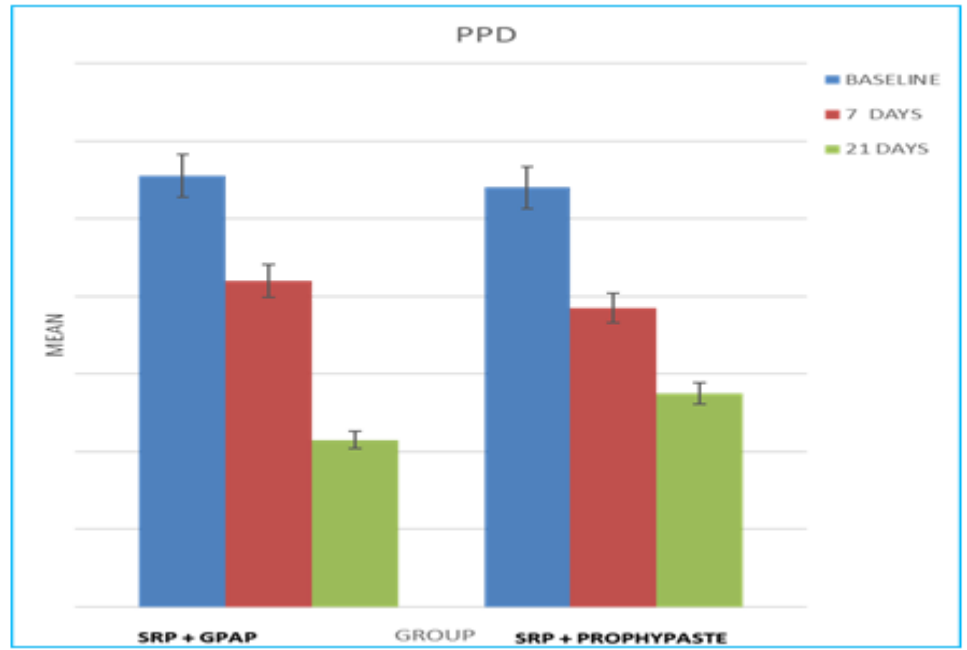

Fig. 5: Graph showing comparison of pocket probing depth test and control group

\section{Discussion}

Periodontal disease involves systemic and initial treatment phases, followed by corrective and long- term supportive-care phases. Scaling and root planing combined with effective plaque control is the gold standard for tackling periodontal infection in the initial treatment phase. Various studies were conducted on comparison of the effectiveness of ultrasonic scaling and hand scaling for removal of subgingival plaque and calculus shows that a large percentage of the treated proximal root surfaces retain stainable deposits, and microbial colonies of plaque smaller than $0.5 \mathrm{~mm}$ remain on the treated tooth surfaces. ${ }^{13}$

Glycine powder air-polishing (GPAP) is an additional approach to nonsurgical periodontal treatment. Using this technique for supportive periodontal therapy, the abrasiveness of different powders is compared with respect to GPAP. Minor erosion of gingival epithelia is seen with GPAP, as compared to sodium bicarbonate powder, which displays moderate to severe erosion immediately after treatment. ${ }^{14}$ In the present study, the Test site was treated with GPAP immediately after SRP, and shown that none of the patients reported any major postoperative pain, discomfort or other complications, except for tooth sensitivity which was reported by some subjects following SRP.

GPAP has been found efficacious in the treatment of periodontal pockets and in the removal of subgingival biofilm. ${ }^{15}$ Marsh et al stated that unresolved inflammation is recognized to be crucial for the progression of periodontal disease as it leads to the growth of micro environmental conditions and affects the proliferation of certain periodontal pathogens. Ji YJ et al stated that GPAP serve as a useful tool in controlling periodontal inflammation and peri-implant disease. In a recently published clinical trial, it has been shown that GPAP may be as effective in subgingival biofilm removal as curettes or ultrasonic scalers in periodontal pockets with probing depths up to approximately $4 \mathrm{~mm} .{ }^{16}$

The strength of the present study depends on the blinding of operators as well as negligible increase in chairside time. Microbiological and histological study could have be done to check the reduction of bacteria and the amount of tissue damage respectively. ${ }^{17-20}$ 


\section{Conclusion}

Within the limitations of this study it is concluded that GPAP, as an additional approach to nonsurgical periodontal treatment, may be beneficial in the shortterm improvement of subclinical periodontal inflammation and it was effective in decreasing inflammation, and pocket probig depth in chronic periodontitis patients and can be successfully used as an adjunct to SRP in routine to non-surgical periodontal therapy. Further investigations with refined clinical protocols and larger sample sizes are needed to determine the exact benefits of GPAP for controlling periodontal disease and maintaining long- term periodontal health.

\section{Conflict of Interest: None.}

\section{References}

1. Petersilka G, Faggion CM Jr, Stratmann U, Gerss J, Ehmke B, Haeberlein I, et al. Effect of glycine powder air-polishing on the gingiva. $J$ Clin Periodontol 2008;35(4):324-32.

2. Petersilka GJ, Bell M, Haberlein I, Mehl A, Hickel R, Flemmig TF et al. In vitro evaluation of novel low abrasive air polishing powders. J Clin Periodontol 2003;30(1):9-13.

3. Kozlovsky A, Artzi Z, Nemcovsky CE, Hirshberg A. Effect of air- polishing devices on the gingiva: histologic study in the canine. J Clin Periodontol 2005;32(4):32934.

4. Petersilka GJ, Steinmann D, Haberlein I, Heinecke A, Flemmig TF. Subgingival plaque removal in buccal and lingual sites using a novel low abrasive air- polishing powder. J Clin Periodontol 2003;30(4):328-33.

5. Flemmig TF, Hetzel M, Topoll H, Gerss J, Haeberlein I, PetersilkaG. Subgingival debridement efficacyof glycine powder air polishing. J Periodontol 2007;78(6):1002-10.

6. Flemmig TF, Petersilka GJ, Mehl A, Ru "diger S, Hickel $\mathrm{R}$, Klaiber B. Working parameters of a sonic scaler influencing root substance removal in vitro. Clin Oral Investig 1998;25(2):158-63.

7. Flemmig TF, Petersilka GJ, Mehl A, Hickel R, Klaiber B. The effect of working parameters on root substance removal using a piezoelectric ultrasonic scaler in vitro. $J$ Clin Periodontol 1998;25(2):158-63.

8. Flemmig TF, Petersilka GJ, Mehl A, Hickel R, Klaiber B. Working parameters of a magnetostrictive ultrasonic scaler influencing root substance removal in vitro. $J$ Periodontol 1998;69(5):547-53.
9. Zappa U, Smith B, Simona C, Graf H, Case D, Kim W. Root substance removal by scaling and root planing. $J$ Periodontol 1991;62(12):750-54.

10. Petersilka G, Faggion CM Jr, Stratmann U. Effect of glycine powder air-polishing on the gingiva. J Clin Periodontol 2008;35(4):324-32.

11. Loe H, Silness J. Periodontal disease in pregnancy I prevalence and severity. Acta Odontol Scand 1963;21:533-51.

12. Turesky S, Gilmore ND, Glickman I. Reduced plaque formation by the chloromethylene analogue of Vitamin C. J Periodontol 1970;41(1):41-3.

13. Breininger DR, O'Leary TJ, Blumenshine RV. Comparative effectiveness of ultrasonic and hand scaling for the removal of subgingival plaque and calculus. $J$ Periodontol 1987;58(1):9-18.

14. Flemmig TF, Arushanov D, Daubert D, Rothen M, Mueller G, Leroux BG. Randomized controlled trial assessing efficacy and safety of glycine powder air polishing in moderate- to- deep periodontal pockets. $J$ Periodontol 2012;83(4):444-52.

15. Caygur A, Albaba RM, Berberoglu A, Yilmaz GH. Efficacy of glycine powder air-polishing combined with scaling and root planing in the treatment of periodontitis and halitosis: A randomised clinical study. J Int Med Res 2017; 45(3):1168-1174.

16. Braun A, Krause F, Hartschen, V, Falk W, Jepsen, S. Efficiency of the Vector system compared with conventional subgingival debridement in vitro and in vivo. J Clin Periodontol 2006 33(8):568-74.

17. Kontturi-Narhi V, Markkanen S, Markkanen H. The gingival effects of dental airpolishing as evaluated by scanning electron microscopy. J Periodontol 1989;60(1):19-22.

18. Kozlovsky A, Artzi Z, Nemcovsky CE, Hirshberg A. Effect of air-polishing devices on the gingiva: histologic study in the canine. J Clin Periodontol 2005;32(4):32934.

19. Patil S, Purshottam S. Rakhewar, Limaye P, Chaudhari N. A comparative evaluation of plaque-removing efficacy of air polishing and rubber-cup, bristle brush with paste polishing on oral hygiene status: A clinical study. J Int Soc Prev Community Dent 2015;5(6):457-62.

20. Newman PS, Silverwood, RA, Dolby AE. The effects of an abrasive instrument on dental hard tissues, skin and oral mucosa. Br Dent J 1985;159:9-12.

How to cite the article: Sharma A, Nair MM, Achom M, Pal C, Sethi M, Sharma N. Comparative evaluation of the efficacy of glycine powder air polishing with scaling and root planing and with fluoride prophypaste as an adjunctive to surgical periodontal therapy-A clinical study. J Dent Specialities 2018;6(2):140-144. 